\title{
Sémiologie/sémiotique chez Saussure et Jakobson: concepts, filiation, débats ${ }^{1}$
}

\section{Semiology/semiotics in Saussure and Jakobson: Concepts, influence, debates}

\author{
Thomas F. Broden \\ Purdue University
}

\begin{abstract}
It was by developing phonology according to the principles of the CGL that the Prague Linguistic Circle succeeded in having linguistics recognized as the most advanced among the human sciences for several decades. The Czech group thereby helped establish Ferdinand de Saussure as a key seminal figure for the modern era, alongside such thinkers as Freud, Marx, and Nietzsche. Roman Jakobson made a particularly significant contribution to the spread of Saussurism, thanks to his genius and his abundant scholarly production, to his ample views and his long, varied career. The first part of this essay briefly recounts key steps in Saussure's elaboration of the idea of the semiological. The second part examines how Jakobson adopted the Saussurian thesis while also adding his own semiotic ideas that amplify, develop, and at times contest certain CGL proposals. This article focuses on the sign and semiosis, or the action of producing signs, and traces the progressive growth of the semiotic domain first in Saussure, than in Jakobson. Having made the choice to sketch a complex and extensive evolution in the two linguists, we are forced to accept that the essay cannot examine in detail each fundamental concept in play.

KEYWORDS: Jakobson; Semiology; Saussure

\section{RÉSUMÉ}

C'est en développant la phonologie selon les principes sémiologiques du Cours de linguistique générale (CLG) que le Cercle linguistique de Prague a réussi à imposer la linguistique comme étant la plus avancée des sciences humaines pendant des décennies. Ce faisant, le groupe tchèque a aidé à faire reconnaître Ferdinand de Saussure comme maître à penser pour l'ère moderne, à côté de figures telles que Freud, Marx et Nietzsche. Roman Jakobson en particulier a beaucoup contribué au rayonnement du saussurisme, par son génie et sa production scientifique abondante, par ses vues généreuses et sa longue carrière variée. Dans une première partie, cet essai retracera brièvement quelques étapes dans l'élaboration de l'idée du sémiologique chez Saussure. Dans la deuxième partie, nous verrons comment Jakobson a repris la thèse saussurienne tout en élaborant ses propres idées sémiotiques qui complètent, développent et contestent parfois certaines propositions du CLG.
\end{abstract}

MOTS-CLÉS: Jakobson; Saussure; Sémiologie

\section{INTRODUCTION}

C'est en développant la phonologie selon les principes sémiologiques du Cours de linguistique générale (CLG) que le Cercle linguistique de Prague a réussi à imposer la linguistique comme étant la plus avancée des sciences humaines pendant des décennies. Ce faisant, le groupe tchèque a aidé à faire reconnaître Ferdinand de Saussure comme maître à penser pour l'ère moderne, à côté de figures telles

${ }^{1}$ Nous remercions Daniele Gambarara et José Luis Fiorin pour leurs commentaires sur une première version de ce texte.

Revista do GELNE, Natal/RN, Vol. 19 - Número Especial/Dossiê: p. 237-247. 2017 
que Freud, Marx et Nietzsche. Roman Jakobson en particulier a beaucoup contribué au rayonnement du saussurisme, par son génie et sa production scientifique abondante, par ses vues généreuses et sa longue carrière variée. Ce savant russe a transformé la linguistique, la poétique, la traductologie et la sémiotique; il a beaucoup fait pour faire triompher le structuralisme au milieu du siècle dernier - et a pris part au lancement des sciences cognitives aussi.

Dans une première partie, cet essai retracera brièvement quelques étapes dans l'élaboration de l'idée du sémiologique chez Saussure. On sait que selon le linguiste, pour comprendre ce qu'est un idiome, il faut le concevoir comme un système et une institution sémiologiques. Dans la deuxième partie, nous verrons comment Jakobson a repris la thèse saussurienne tout en élaborant ses propres idées sémiotiques qui complètent, développent et contestent parfois certaines propositions du CLG. En effet, Jakobson partageait l'attitude de Benveniste envers ce texte, telle qu'Irène Fenoglio l'expose dans "La langue et l'écriture": un écart théorique entre Benveniste et Saussure (aussi dans ce numéro). Il a considéré le CLG comme un ouvrage génial et fondateur qu'il ne fallait pas répéter comme un texte sacré, mais développer dans un esprit scientifique. Nous nous concentrerons sur les concepts du signe et de la sémiosis, ou l'action de produire des signes, et décrirons comment le champ du sémiotique s'est élargi progressivement d'abord chez Saussure, ensuite chez Jakobson. Ayant fait le choix de dresser un déroulement complexe et important chez ces deux savants, nous devons accepter l'inconvénient de ne pouvoir creuser en détail chaque concept fondamental abordé, ce qu'il faudrait bien sûr faire en principe.

\section{Le développement du sémiologique chez Ferdinand de Saussure}

Saussure a couché sur papier certaines idées sur la sémiologie au cours de sa carrière lorsqu'il a abordé de différentes problématiques, écrits restés inédits de son vivant. À l'âge de 26 ans, trois années après avoir soutenu sa thèse, le linguiste genevois a préconisé que les sons d'une langue se définissent par la convergence de trois perspectives: "Délimitation au nom de la sémiologie du phonème (négative seulement) [...] Délimitation acoustique du phonème $[. .$.$] Délimitation des causes$ physiologiques du phonème" (PARRET, 1993, p. 203). Dans ce brouillon de 1883-1884, "la sémiologie", terme peu usité alors en dehors de la médecine où il désigne la symptomatologie, sert à désigner la constitution négative des unités sonores. La citation affirme qu'il faut tenir compte de données relevant de trois instances pour définir tout son linguistique: celles de son architecture oppositive, de l'énonciataire et de l'énonciateur. Le même texte insiste sur l'armature solidaire qui forme les sons (comme venait de le démontrer le Mémoire sur le système primitif des voyelles dans les langues indo-européennes): "Le langage se compose d'un système d'oppositions acoustiques »(SAUSSURE, 1878).

Dix ans après, un inédit de Saussure sur la linguistique générale étend cette conception d'un ensemble différentiel à la sémiosis, à la production des signes, et non plus aux seuls sons. De l'essence double du langage s'oppose à la doxa qui conçoit le signe comme une entité binaire isolée composée d'un son et d'un concept : il existe "seulement en réalité des différences de formes et des différences de significations ; [...] négatives en elles-mêmes », telles que " chacun de ces ordres [...] n'existe comme différences que grâce à l'union avec l'autre ; [...] cette opposition de valeur qui est un fait PUREMENT NÉGATIF se transformera en fait positif, parce que chaque signe [...] se trouve être délimité, malgré nous, dans sa valeur propre » (SAUSSURE, 2002, p. 42-3; 87-8). Ce passage résume les concepts clés du CLG que sont la valeur, la définition mutuelle des signifiants et des signifiés, et la transformation des signifiants et des signifiés négatifs en signes positifs.

Le brouillon de 1891-1894 sur la linguistique générale insiste sur le caractère dynamique du

langage. À côté du "point de vue instantané», le "point de vue diachronique » ou « historique » décrit

Revista do GELNE, Natal/RN, Vol. 19 - Número Especial/Dossiê: p. 237-247. 2017 
l'évolution d'un idiome au cours du temps (SAUSSURE, 2002, p.21-22; 50). De même, dans une conférence qu'il a donnée à l'Université de Genève en 1891, Saussure a souligné « la mutabilité » de la langue, le "mouvement de la langue dans le temps [...: :] il y a transformation, et toujours et encore transformation, [...] il n'y a jamais en réalité un équilibre, un point permanent, stable dans aucun langage » (2002, p. 157-8). D'autre part, De l'essence double du langage explique aussi que le langage luimême ne consiste qu'en opérations, en processus, alors que l'esprit se trouve obligé de traduire ceuxci en «substance» pour les penser : «SUBSTANCE LINGUISTIQUE. - Nous tendons perpétuellement à convertir par la pensée en substance les actions diverses que nécessite le langage. Il semble nécessaire dans la théorie même d'épouser cette conception » (SAUSSURE, 2002, p. 81). Toute description statique des unités linguistiques représente donc une déformation malheureuse mais inéluctable du phénomène dynamique réel.

Les cours sur la linguistique générale que Saussure a assurés à Genève une quinzaine d'années plus tard (GODEL, 1957) reprennent et développent sa conception de la langue comme système solidaire unique (SAUSSURE, 1993). Les conférences de 1906-1911 qui donneront lieu au CLG précisent que pour définir le signe linguistique, il faut partir de l'ensemble dont il fait partie, et non vice-versa : "Il ne faut pas commencer par le mot, le terme, pour en déduire le système. Ce serait se figurer que les termes ont d'avance une valeur absolue, qu'il n'y a qu'à les échafauder les uns sur les autres pour avoir le système. Au contraire, c'est du système, du tout solidaire qu'il faut partir » (SAUSSURE, 1993, p.134). En synchronie, le "mécanisme de la langue » ajoute dorénavant la combinaison au différentiel: tout en s'opposant "in absentia» sur le plan "associatif», les unités s'unissent et se disposent aussi « in praesentia » sur le plan « syntagmatique ».

Dans les recherches sur les épopées germaniques qu'il a poursuivies vers la même époque, de juin 1903 à avril 1911, Saussure a étendu sa conception sémiologique à d'autres phénomènes discursifs. Ses notes sur les légendes affirment que les personnages se définissent comme des « symboles », c'està-dire des unités sémiologiques régies par les mêmes principes qui gouvernent les signes linguistiques:

La légende se compose d'une série de symboles [...qui] sont soumis aux vicissitudes et aux mêmes lois que toutes les autres séries de symboles, par exemple les symboles qui sont les mots de la langue. Ils font tous partie de la sémiologie. [...] L'identité d'un symbole ne peut jamais être fixé depuis l'instant où il est symbole, c'est-à-dire versé dans la masse sociale qui en fixe à chaque instant la valeur. [...] chacun des personnages est un symbole dont on peut voir varier [...] a) le nom, b) la position vis-à-vis des autres - c) le caractère ; d) la fonction, les actes (SAUSSURE, 1985, p. 30).

Les brouillons mettent en relief avant tout la plasticité des symboles-personnages, dont la valeur se transforme sur la dimension diachronique dans la perspective de l'arbitraire du signe qui est désormais au cœur de la sémiologie saussurienne. De ces personnages, sont sujets au changement leur signifiant, leur signifié - et cela sur deux plans, l'être et le faire du personnage - et la relation aux autres personnages des légendes.

Dans la citation sur les symboles de même que dans ses cours de linguistique générale, Saussure a posé la sémiologie comme une nouvelle discipline autonome, qu'il définit : «[1]a sémiologie (étude des signes et de leur vie dans les sociétés humaines)» (SAUSSURE, 1993, p. 71), "science [qui] ne s'est pas encore imposée» (GODEL, 1957, p. 67), mais qui embrasse «[t]out un ordre de faits psychologiques (de psychologie sociale) qui ont droit d'être étudiés comme un seul ensemble de faits » (SAUSSURE, 1993, p. 71). On peut chercher les lois qui régissent de tels ensembles, qui présentent 
par exemple des particularités constantes de caractère à la fois interne et externe : tout signe y est «arbitraire» et «purement négatif et différentiel», et si «le système suppose une convention ", «l'individu ou la communauté ne peuvent ni le modifier ni l'empêcher d'évoluer» $»($ GODEL, 1957, p. 66).

Les cours sur la linguistique générale élargissent la nouvelle science des signes en incorporant des pratiques non-linguistiques et définissent tout système sémiologique comme une institution sociale : «Il faudrait donc faire entrer la langue dans les institutions sémiologiques; celle des signaux maritimes par exemple (signes visuels), les signaux de trompettes militaires, le langage des sourdsmuets, etc. L'écriture est également un vaste système de signes » (SAUSSURE, 1967, p. 47). Le passage rappelle qu'à la suite de $\mathrm{D}$. Whitney, les conférences ont insisté sur le caractère social et conventionnel de la langue, et par extension des autres ensembles sémiologiques. Certaines de ces pratiques affichent une gestualité codifiée et « arbitraire », telles que les « signes de politesse » ou « le geste par lequel nous saluons » (SAUSSURE, 1967, p. 47); «[u]ne cérémonie, un ensemble de rites sont des institutions rappelant un peu la langue »(SAUSSURE, 1967, p. 46). Pour Saussure, c'est grâce au sémiologique que le linguiste peut situer sa discipline parmi les sciences.

Lorsqu'il présente la dimension sonore du langage dans ses cours de linguistique générale, Saussure reprend et développe ses brouillons de 1883-1884 en observant que la description des unités phoniques doit tenir compte des perspectives de l'énonciateur et de l'énonciataire, de même que du système de signes où s'associent les sons et les concepts (GODEL, 1957). Les cours et De l'essence double du langage mettent en avant parfois l'acte unique de langage à côté du tout solidaire et insistent sur la complémentarité de ces deux dimensions du langage que sont le système et l'événement ${ }^{2}$. On sait que les éditeurs du CLG ont gommé bien des hésitations et des ambivalences du professeur pour mettre plus clairement à l'honneur la langue aux dépens de la parole. De toute façon, dans les notes prises par les étudiants de Saussure comme dans le CLG, ni l'énonciateur ni l'énonciataire ne sont mis en évidence dans les expositions centrales sur le signe, la valeur et les unités de la langue; le système solidaire, souvent présenté sous forme d'une topologie, y occupe à lui seul toute la place. Le « circuit de la parole » où « deux personnes » «s'entretiennent » sert à préciser le site où définir et étudier la langue, mais s'éclipse aussitôt (SAUSSURE, 1993, p. 67). Le CLG néglige ainsi l'action du sujet parlant et la communication intersubjective ou sociale pour insister davantage sur l'architecture que forment les signes. Saussure indique toutefois que l'énonciateur reprendrait un rôle important si l'on étudiait la syntaxe, délaissée aux dépens de la dimension phonique du langage (SAUSSURE, 1993).

De la même façon, au tout début des conférences consacrées à la linguistique générale en 19101911, Saussure a expliqué que la conception commune de la langue comme "nomenclature d'objets » reste critiquable, qu' "il y a bien un objet qui est hors du sujet, et le nom », mais que «le lien entre les deux n'a rien de clair» (SAUSSURE, 1967, p. 148). On peut supposer que cet objet joue un rôle dans l'observation que «le signifié 'bœuf a pour signifiant $b$ - $\ddot{o}-f$ d'un côté de la frontière et $o-k-s(O c h s)$ de l'autre » (SAUSSURE,1972, p. 100). Logiquement, la dernière partie du cours qui devait examiner «l'exercice du langage chez les individus » aurait pu reprendre cette question pour explorer le lien entre l'objet et le nom dans la parole, sujet pertinent s'il en est dans l' « étude des signes et de leur vie dans les sociétés humaines » (SAUSSURE, 1993, p. 71). Malheureusement, les grandes vacances sont arrivées avant que le professeur n'ait pu terminer la deuxième partie du cours et en aborder la troisième.

\footnotetext{
${ }^{2}$ Voir par exemple : «Tout ce qui entre dans la langue a d'abord été essayé dans la parole un nombre de fois suffisant pour qu'il en résulte une impression durable ; la langue n'est que la consécration de ce qui avait été évoqué < par > la parole », Riedlinger, notes du deuxième cours, cité in SAUSSURE (1967, p. 376 et aussi SAUSSURE (1972, p. 37).
} 
Afin de rendre sensible ce qu'est «un système serré comme la langue» en décrivant «la multiplicité immense des termes ou unités du système, leur stricte dépendance réciproque» (GODEL,1957, p. 71), les exposés des cours insistent beaucoup sur l'illustration topologique, comme l'attestent les nombreux diagrammes que l'on trouve dans les notes de cours et le CLG. En effet, pour parler des signes linguistiques, Saussure a quasi-systématiquement recours à des formulations qui identifient ou posent des formes spatiales, telles que: «Là est le terrain de la linguistique, celui des articuli... le caractère linéaire de la matière phonique oblige d'abord de découper celle-ci pour avoir des unités »(GODEL, 1957, p. 68); "La délimitation formera des chaînons sur une même ligne » (SAUSSURE, 1993, p. 80); "L'unité est ainsi une tranche de sonorité... liée à un certain concept qui sert à délimiter la tranche » (soulignements dans le texte) (GODEL, 1957, p. 83). De même, chaque signe ainsi défini prendra la forme d'un objet plan à « recto » et « verso » (phonique et conceptuel) (SAUSSURE, 1972, p. 157).

En même temps, en nous rappelant le passage dans De l'essence double du langage sur la substance linguistique, il faut garder à l'esprit que si ces figures et formules topologiques qui servent d'illustrations didactiques se présentent comme des objets inertes, elles pointent des processus dynamiques. En effet, on sait que si Saussure a fait ses études doctorales chez les néogrammairiens à Leipzig, à Paris il s'est rallié à la position de Bréal que la langue est un dynamisme cognitif vivant. Les cours de linguistique générale décrivent cette créativité linguistique lorsqu'ils étudient le fonctionnement de l'analogie, les relations associatives, l'étymologie populaire et la propagation des innovations linguistiques. Ces phénomènes mettent en relief le langage en tant qu' "activité continuelle » (SAUSSURE, 1967, p. 376), " processus générateur », ensemble de « formes génératrices »; "La langue ne cesse d'interpréter et de décomposer les unités qui lui sont données » (SAUSSURE, 1972, p.227). Dans une sémiosis constante et sans fin, l'esprit reprend continûment les signes, les réanalyse et les refait : « < la langue $>$ passe son temps à interpréter et à décomposer ce qui est en elle l'apport des générations précédentes [...] pour ensuite avec les sous-unités qu'elle a obtenues combiner de nouvelles constructions. [...] La langue recompose $<$ dans la mesure où $>$ elle décompose et elle sera d'autant plus créatrice dans la mesure où elle aura plus de matériaux »(SAUSSURE, 1967, p. 386). Chaque communauté et individu reprennent ou récusent toute innovation ou écart pour l'incorporer dans l'idiome ou l'en éliminer.

\section{La place du CLG chez le Cercle linguistique de Prague et Roman Jakobson}

La phonologie élaborée par le Cercle de Prague selon les principes du CLG a fini par imposer la linguistique comme «science pilote » pour d'autres sciences humaines, en attirant l'émulation de C. Lévi-Strauss, J. Lacan, R. Barthes, L. Althusser et d'autres chercheurs de pointe. Or, dès les premières pages de Grundzüge der Phonologie, dont la traduction française Principes de phonologie a servi de manuel pour Lévi-Strauss et bien d'autres, N. Troubetzkoy situe l'approche de son œuvre fondatrice dans les perspectives du CLG. Pour formuler sa distinction centrale entre la phonologie et la phonétique, il fait appel à la définition de la langue et de la parole, à l'idée du signe constitué par une relation privilégiée entre le signifiant et le signifié et à la conception de la langue comme système ${ }^{3}$. Le livre développe de façon systématique la thèse saussurienne que la langue représente non pas une substance mais une forme constituée par des relations.

\footnotetext{
${ }^{3}$ "La différence existant entre "parole" et "langue" fut d'abord reconnue de la façon la plus nette par le linguiste suisse Ferdinand de Saussure, dans son "Cours de linguistique générale" [...]. Tout ce qui appartient au langage, c'est-à-dire aussi bien acte de parole que langue, a d'après Ferdinand de Saussure deux faces : le signifiant et le signifié, de sorte qu'un langage est toujours une association, un recouvrement réciproque du "signifiant" et du "signifié" [...] la langue est un système, ou, plus, précisément un ensemble de systèmes partiels » (TROUBETZKOY, 1949, p. 2-3).
} 
De même, Jakobson a repris et développé des principes importants du CLG à Moscou, à Prague, en Scandinavie et aux États-Unis. En 1942, l'exilé russe a assuré par exemple un «Cours sur la doctrine linguistique de Ferdinand de Saussure » à l'École libre des Hautes Études à New York (JAKOBSON, 1984), auquel ont assisté Lévi-Strauss et de jeunes étudiants en linguistique tels que Paul Garvin, Charles Hockett et Thomas A. Sebeok (WAUGH, 1984) ${ }^{4}$. Les notes que Jakobson a composées pour ce cours indiquent que la première partie a discuté en détail la conception de la langue et de la parole tandis que la deuxième partie a traité le signe linguistique et le phonème. Le linguiste russe a affirmé à propos du CLG qu' "aucun autre livre de notre siècle n'a exercé une influence si large et profonde sur la linguistique internationale ; [...] Presque tous les problèmes essentiels de la linguistique moderne se trouvent ébauchés dans ce livre » (JAKOBSON, 1984, p. 165).

En même temps, dans son cours à New York, Jakobson a observé à propos du CLG que « ce ne sont donc pas des dogmes définitifs, mais des hypothèses de travail et des esquisses lucides » (JAKOBSON, 1984, p. 164). En effet, le cours de 1942 a exposé les principes du CLG, mais a aussi proposé des amendements et des critiques importants. Le savant russe a notamment souligné que «Saussure n'a pas déployé une théorie conséquente du facteur intersubjectif dans le langage », et a cité Voloshinov en insistant sur l'importance du dialogue et de la dynamique d'échange, d'interaction et d'ajustement au sein de l'entretien (JAKOBSON, 1984, p. 177). On sait que Jakobson a repris mais aussi repensé les concepts de la synchronie et de la diachronie, de la langue et de la parole et du principe de l'arbitraire. Dans certains cas, il a généralisé des éléments du CLG en étendant leur application à d'autres champs linguistiques, et parfois aux formes d'expression non-linguistiques. Son article traduit en français comme «Deux aspects du langage et deux types d'aphasie » développe ainsi les relations associatives et syntagmatiques en montrant que les opérations de la sélection et de la combinaison distinguent deux phases de l'acquisition du langage, déterminent deux types contrastés d'aphasie, soustendent la métaphore et la métonymie en rhétorique et informent le symbolisme et le réalisme respectivement dans les domaines de l'art plastique et de la littérature (JAKOBSON, 1963). Jakobson a publié des travaux linguistiques importants sur la poétique, la littérature et le folklore, champs qui occupaient bien des recherches de Saussure, sans que celui-ci ait mené ces investigations à terme pour une publication. La vision large et généreuse de la linguistique chez le savant russe s'oppose à la concentration presque exclusive sur la langue que l'on constate dans le CLG; on sait qu'il aimait revendiquer son adaptation de la formule de Térence, "Je suis linguiste, rien de ce qui touche au langage ne m'est étranger». De manière générale, les développements de Jakobson vont vers l'expansion et la complexité, alors que dans ses cours, Saussure s'est efforcé de condenser et de simplifier. Jakobson a même visé l'universel davantage que Saussure, qui a préféré le général et le comparatif.

\section{Le développement du sémiotique chez Jakobson}

Dans ses recherches qui portent de façon directe sur la sémiotique, Jakobson a traité de la sémiologie saussurienne, de la sémantique chez les philosophes et les linguistes du vingtième siècle et de la grammaire spéculative médiévale. Mais c'est C. S. Peirce qu'il a mis le plus en avant en discutant de la sémiotique. Il a découvert les écrits sur le signe du philosophe américain dès le début des années 1950 et n'a cessé de les exploiter dans des travaux rédigés ou traduits en français qui élaborent des éléments de sémiotique. Peirce servait de référence stratégique à Jakobson qui évoluait aux ÉtatsUnis et qui voulait ouvrir la linguistique post-bloomfieldienne à la réflexion théorique : le philosophe

${ }^{4}$ Et l'année suivante, il a eu Mattoso Câmara comme étudiant à Columbia University, comme nous le précise José Luiz Fiorin.

Revista do GELNE, Natal/RN, Vol. 19 - Número Especial/Dossiê: p. 237-247. 2017 
américain véhicule la métaphysique classique, mise à jour dans un contexte de logique et de références aux sciences dures susceptible de séduire en Amérique.

Dès 1952, dans une conférence reprise dans le premier tome des Essais de linguistique générale parus en 1963, le linguiste russe a présenté le sémioticien américain comme "un des plus grands précurseurs de l'analyse structurale en linguistique », dans la mesure où il a non seulement «établi la nécessité de la sémiotique» comme Saussure, mais "en a aussi esquissé les grandes lignes» (JAKOBSON, 1963, p. 27). Cette sémiotique peut être utile aux linguistes, car même si « le système sémiotique le plus important, la base de tout le reste, c'est le langage [...] tous les autres systèmes de symboles sont accessoires ou dérivés ", comparer les langues aux autres systèmes symboliques révèle les principes essentiels du langage, et permet de déterminer ses traits spécifiques (JAKOBSON, 1963, p. 28). On voit que s'il cite Peirce, Jakobson retrouve aussi la perspective de Saussure, phénomène qu'on trouve souvent dans ses discussions sur la sémiotique.

En 1953, Jakobson met en relief le concept peircien de l'interprétant, l'idée que « tout signe se traduit dans un autre signe », pour défendre le projet d'une sémantique linguistique immanente, à l'encontre de la sémantique élaborée par la philosophie analytique qui privilégie le référent nonlinguistique. En effet, l'interprétant communique bien l'attitude épistémologique de Peirce, selon qui «Tout cet univers est imprégné de signes, sinon composé exclusivement de signes ». L'interprétant permet à Jakobson « d'insister sur le caractère intrinsèquement linguistique de la sémantique »:

Dès 1867, C.S. Peirce, qui, je le répète, doit être considéré comme un authentique et intrépide précurseur de la linguistique structurale, avait nettement établi le caractère linguistique de la sémantique. [...] Peirce donne une définition incisive du principal mécanisme structural du langage quand il montre que tout signe peut être traduit par un autre signe dans lequel il est plus complètement développé (JAKOBSON, 1963, p. 41).

La formule de Jakobson reprend la définition de la sémiosis que le sémioticien américain a élaborée vers 1897, soit quelques années seulement après que Saussure a exposé sa conception de la langue comme un système de signes dans De l'essence double du langage:

Un signe, ou representamen, est quelque chose qui tient lieu pour quelqu'un de quelque chose sous quelque rapport ou à quelque titre. Il s'adresse à quelqu'un, c'est-à-dire crée dans l'esprit de cette personne un signe équivalent ou peut-être un signe plus développé. Ce signe qu'il crée, je l'appelle l'interprétant du premier signe. Ce signe tient lieu de quelque chose: de son objet (PEIRCE, 1978, p. 121).

Chez Peirce, le terme de representamen ou « signe » correspond à ce que la linguistique et la sémiotique romanes désignent comme « discours » et « texte ». L' « objet » peircien se situe pleinement dans sa phénoménologie, à l'encontre de la philosophie du langage formaliste et logiciste. Cet objet, «ce dont la connaissance est présupposée pour pouvoir communiquer des informations supplémentaires le concernant » (PEIRCE, 1978, p. 123), a donc surtout pour mission d'introduire le contexte à côté du texte, qu'il s'agisse du contexte énoncif, "interne », ou du contexte énonciatif, « externe ». Peirce indique par exemple que l'objet d'un pronom relatif comme que ou qui est son antécédent grammatical, son référent interne, discursif (PEIRCE, 1978, p. 155-56). 
La définition citée pose un processus que le philosophe américain appelle ailleurs la sémiosis et qu'on peut comparer sous certains aspects à l'énonciation. Dans cette définition, le representamen s'adresse à l'énonciataire et suscite chez celui-ci un nouveau signe. En transposant la terminologie de façon approximative (et abusive), on peut dire que le signifiant génère un signifié chez un sujet déterminé dans un contexte particulier. Alors que dans le positivisme logique que combattait Jakobson, le «signe » ou signifiant isolé s'associe à un concept mental non-linguistique dont le sens dépend du référent situé dans le monde naturel et garanti par la science, chez Peirce, tous les composants de la sémiosis relèvent du fonctionnement sémiotique. Par contre, n’en déplaise au linguiste russe, l'interprétant peircien n'est pas forcément linguistique; qu'il le soit ou non indiffère au philosophe américain. D'autre part, on voit que Peirce définit la sémiosis dans le champ de la parole saussurienne plutôt que dans celui de la langue, et vise à articuler non pas une topologie de signes mais plutôt un acte humain signifiant.

La conception saussurienne et celle peircienne de la sémiosis se complètent plutôt qu'elles ne s'opposent. Saussure insiste à juste titre sur l'organisation complexe des ensembles signifiants tels qu'une langue ou un énoncé et sur la spécificité socio-historique d'un système sémiologique à chaque moment de son développement. La formulation peircienne enrichit celle saussurienne en élargissant son champ et en mettant d'emblée en avant bon nombre de problématiques clés de la linguistique et de la sémiotique, dont notamment l'énonciation, la temporalisation, l'acte, le contexte et l'interaction entre pratiques de substance différente.

En 1958, Jakobson a présenté «les facteurs constitutifs de tout procès linguistique, de tout acte de communication verbale », au nombre de six: le destinateur, destinataire, message, code, contact et contexte. À chaque composante correspond une fonction linguistique distincte : expressive, conative, poétique, métalinguistique, phatique et référentielle (JAKOBSON, 1963). On sait que Jakobson n'a pas cherché à donner à son modèle une formulation hautement théorique, et qu'on a pu critiquer le choix, la définition et l'articulation de ses composantes inspirées de Bühler et de la théorie de l'information. Nous nous contentons de retenir que ce schéma incorpore la langue saussurienne sous forme du «code », mais met sur le même plan d'autres aspects de la communication, y compris le destinateur, le destinataire et la référence. Dans sa pragmatique et dans sa définition du signe citée, Peirce met en avant ces trois dernières instances, que le CLG évoque mais néglige, alors que dans ses définitions du son linguistique, Saussure fait systématiquement appel au destinateur et au destinataire. Le modèle jakobsonien pose aussi la poétique sur un pied d'égalité avec le code, alors que le CLG y fait l'impasse - tandis que les recherches de Saussure sur les anagrammes explorent à fond les mécanismes sémiologiques des procédés prosodiques. Les six facteurs et fonctions possèdent l'avantage insigne de pointer des axes fondamentaux pour la linguistique et la sémiotique qui vont bien au-delà du seul système formel qui domine la sémiosis dans le CLG et du référent dont se préoccupe la philosophie analytique.

Dans son article « Aspects linguistiques de la traduction », Jakobson s'appuie sur l'interprétant peircien pour formuler les principes d'une traductologie et d'une intersémioticité immanentes. « Pour le linguiste comme pour l'usager ordinaire du langage, le sens d'un mot n'est rien d'autre que sa traduction par un autre signe qui peut lui être substitué, spécialement par un autre signe "dans lequel il se trouve plus complètement développé", comme l'enseigne Peirce, le plus profond investigateur de l'essence des signes » (JAKOBSON, 1963, p. 79). En effet, Peirce lui-même évoque souvent le processus de «traduction » au cœur de la signification : « un signe n'est pas un signe à moins qu'il ne se traduise dans un autre signe dans lequel il est plus développé »; «le sens [... est] dans son acceptation primaire la traduction d'un signe dans un autre système de signes »; «le sens d'un signe est le signe dans lequel il doit être traduit»(LISZKA, 1966, p. 24). À côté de la traduction 
« intralinguale » ou « reformulation » au sein d'une même langue et de la traduction « interlinguale » ou traduction proprement dite, Jakobson introduit la notion de «traduction intersémiotique ou transmutation ", à savoir « l'interprétation des signes linguistiques au moyen de systèmes de signes non linguistiques [...] par exemple de l'art du langage à la musique, à la danse, au cinéma ou à la peinture » (JAKOBSON, 1963, p. 79). En citant Bertrand Russell, le linguiste russe contraste cette perspective immanente de façon explicite avec le point de vue du positivisme logique qui prédomine dans le monde anglo-saxon (1963, p. 79). Remarquons que Jakobson présente ces arts non-verbaux comme des «systèmes de signes », ce qui reprend la conception saussurienne de pratiques sémiologiques multiples et distinctes dont chacune comprend un ensemble complexe et solidaire, alors que Peirce présente une dynamique sémiotique universelle, sans insister sur l'autonomie et le caractère systématique de certains ensembles de signes tels que les langues naturelles ou les traditions culturelles.

Ailleurs, Jakobson reprend la trilogie peircienne icône, indice et symbole pour développer des vues qui complètent ou contestent certaines perspectives du CLG. La définition des shifters dépend du symbole peircien ou de la langue saussurienne, mais aussi de l'indice peircien qui implique l'acte de langage et son contexte (JAKOBSON, 1963). D'autre part, l'article de 1965, "À la recherche de l'essence du langage », montre comment l'icône peircienne permet de relativiser encore plus le principe saussurien de l'arbitraire du signe, en étudiant de multiples structures linguistiques où la configuration du signifié reflète celle du signifiant - phénomènes que la linguistique cognitive a repris depuis (JAKOBSON, 1965).

Dans sa communication qui a inauguré le premier congrès de l'Association Internationale de Sémiotique à Milan en 1974, Jakobson a esquissé une conception large et compréhensive de la sémiotique qui va même au-delà de la sémiologie posée dans le $C L G$ et de la sémiotique philosophique envisagée par Peirce, pour embrasser l'ensemble des recherches sémiotiques en cours dans de multiples domaines non-linguistiques tels que l'image planaire, le cinéma, la musique et la danse recherches qui se réclamaient souvent de la sémiologie saussurienne et du CLG (JAKOBSON, 1979). Cette intervention s'appuie encore une fois sur l'interprétant peircien pour formuler une approche immanente en sémiotique, et insiste que la sémiotique peircienne et la linguistique structurale sont absolument compatibles.

\section{Conclusion : sémiologie saussurienne, sémiotique jakobsonienne et après}

Dans les écrits de Saussure et de Jakobson, le champ sémiotique n'a cessé de s'accroître au cours de la carrière de chaque savant. Le linguiste russe a repris l'idée saussurienne centrale des signes constitués par un système solidaire sujet au changement continu. Il a poursuivi aussi l'ouverture aux pratiques non-linguistiques tout en réaffirmant la spécificité et la primauté des langues naturelles parmi les systèmes de signes. Par rapport au $C L G$, son apport dans le domaine de la sémiotique consiste en premier lieu à insister sur l'acte du langage et de mettre en relief le rôle du contexte, des actants énonciatifs et de la poétique. Dans cette insistance sur l'acte et sur les instances autres que le système interne de la langue, Jakobson retrouve les perspectives pragmatiques et sémiotiques de C. S. Peirce mais aussi celles des inédits de Saussure. Dans ses travaux, le savant russe a aussi pris certaines positions qui vont à l'encontre du CLG, en accordant plus d'importance à la motivation du signe, en revendiquant le caractère téléologique des changements linguistiques et en posant que l'usage individuel manifeste des régularités au même titre que l'usage social.

Par ailleurs, en mettant en relief le contexte de l'acte linguistique ou sémiotique et en accordant l'importance due au destinateur, au destinataire et à la référence, Jakobson rejoint aussi les vues de Benveniste sur le sémantique et l'énonciation, et anticipe sur les conceptions de la sémiosis 
qu'élaboreront A. J. Greimas, Jean-Marie Klinkenberg et François Rastier. Pour compléter la célèbre définition topologique du signe dans le CLG, bon nombre de linguistes et sémioticiens ultérieurs ont ainsi insisté sur quatre thématiques que l'on trouve en germe chez Jakobson et Peirce - et aussi dans les inédits de Saussure: a) l'acte de langage et les pratiques signifiantes ; b) l'interaction, la subjectivité et la communication entre sujets de l'énonciation ; c) l'intentionnalité, voire l'engagement éthique ; le rapport qu'entretient le langage avec le monde et l'histoire.

\section{Références}

GODEL, Robert. Les Sources manuscrites du Cours de linguistique générale de F. de Saussure. Paris, Minard et Genève, Droz, coll. Société de publications romanes et françaises nº 61, 1957.

Greimas, A. J. et Joseph Courtés. Sémiotique. Dictionnaire raisonné de la théorie du langage. Paris, Hachette, 1979.

JAKOBSON, Roman. À la recherche de l'essence du langage. Diogène, n 51, 1965, p. 22-38.

JAKOBSON, Roman. Essais de linguistique générale. Vol. 1. Les fondations du langage. Paris, Minuit, 1963.

JAKOBSON, Roman. Coup d'œil sur le développement de la sémiotique. Actes du premier congrès de l'Association Internationale de Sémiotique, Milan, juin 1974, éd. Seymour Chatman, Umberto Eco et JeanMarie Klinkenberg. La Haye, Mouton, 1979, p. 3-18.

JAKOBSON, Roman. La théorie saussurienne en rétrospection. Linguistics, vol. 22, n² 2, 1984, p. 161-196.

KLINKENBERG, Jean-Marie. Précis de sémiotique générale. Paris, Seuil, 1996.

LISZKA, James. A General Introduction to the Semeiotic of Charles Sanders Peirce. Bloomington, Indiana University Press, 1996.

PARRET, Herman. Les manuscrits saussuriens de Harvard. Cabiers Ferdinand de Saussure, n 47, $1993-$ 1994, p. 179-234.

PEIRCE, Charles Sanders. Collected Papers of Charles Sanders Peirce, éd. Charles Hartshorne et Paul Weiss. 6 vol. Cambridge: Harvard University Press, 1960.

PEIRCE, Charles Sanders. Écrits sur le signe, éd. Gérard Deledalle. Paris, Seuil, 1978.

SAUSSURE, Ferdinand de. Cours de linguistique générale, éd. Rudolf Engler. 2 vol. Wiesbaden, Harrassowitz, 1967.

SAUSSURE, Ferdinand de. Cours de linguistique générale, premier et troisième cours d'après les notes de Riedlinger et Constantin, éd. E. Komatsu. Tokyo, Gakushuin University, 1993.

Revista do GELNE, Natal/RN, Vol. 19 - Número Especial/Dossiê: p. 237-247. 2017 
SAUSSURE, Ferdinand de. De dangereux édifices. Saussure lecteur de Lucrèce. Les "Cahiers d'anagrammes" consacrés au "De rerum natura," éd. F. Gandon. Louvain et Paris, Peeters, coll. Bibliothèque de l'information grammaticale $n^{\circ}$ 50, 2002.

SAUSSURE, Ferdinand de. Écrits de linguistique générale, éd. Simon Bouquet et Rudolf Engler avec Antoinette Weil. Paris, Gallimard, 2002.

SAUSSURE, Ferdinand de. Le leggende germaniche: scritti scelti e annotati, éd. Marcello Meli et Anna Marinetti. Este, Zielo, 1985.

SAUSSURE, Ferdinand de. Mémoire sur le système primitif des voyelles dans les langues indo-européennes. Leipzig, Teubner, 1878.

SAUSSURE, Ferdinand de. Les mots sous les mots. Les anagrammes de Ferdinand de Saussure, éd. Jean Starobinski. Paris, Gallimard, 1971.

SAUSSURE, Ferdinand de. Phonétique : il manoscritto di Harvard Houghton library bMs Fr 266 (8), éd. Maria Pia Marchese. Padoue: Unipress, 1995.

SAUSSURE, Ferdinand de. Troisième cours de linguistique générale (1910-1911) d'après les cabiers d'Emile Constantin, éd. E. Komatsu. Oxford, Pergamon Press, coll. Language and Communication Library $\mathrm{n}^{\circ}$ 12, 1993.

TIERCELIN, Claudine. C. S. Peirce et le pragmatisme. Paris, Presses Universitaires de France, 1993. En ligne, http://books.openedition.org/cdf/1997?lang=en.

TROUBETZKOY, Nikolai Sergeevich. Travaux du cercle linguistique de Praque 7, numéro spécial consacré à Grundzüge der Phonologie, 1939. Traduit par J. Cantineau Principes de phonologie. Paris, Klincksieck, 1949.

WAUGH, Linda. «Introduction ». Linguistics vol. 22, n² 2, 1984, p. 157-160.

WHITNEY, William Dwight. The Life and Growth of Language: An Outline of Linguistic Science. New York, D. Appleton, 1896. 